\title{
Syntactic pattern recognition based on spectrum of spectra
}

\author{
R CHHIBBER $^{1}, \mathrm{~K}^{\mathrm{N}}$ KHATTRI $^{2}$ and P S MOHARIR ${ }^{3}$ \\ ${ }^{\prime}$ Schlumberger (India), Mohan Dev Building, 13, Tolstoy Marg, New Delhi \\ 110001 , India \\ ${ }^{2}$ Wadia Institute of Himalayan Geology, 33, General Mahadev Singh \\ Road, Dehradun 248 001, India \\ ${ }^{3}$ National Geophysical Research Institute, Uppal Road, Hyderabad \\ 500007 , India
}

\begin{abstract}
From Fourier optics it follows that it is possible to obtain a Fourier transform of an amplitude distribution in a transparency readily. In intermediate planes an interpolation between the signal and its Fourier spectrum is available. Some pattern recognition schemes may work better in one of these intermediate domains than in the time or the frequency domain.

A syntactic pattern recognition scheme to achieve seismic discrimination among various hydrocarbon regimes was set up. It used autoregressive parameters as pattern primitives and defined an alphabet based on clusturing in the space of these primitives. Classification was conducted on the basis of nearest neighbour rule using Levenshtein distance between alphabetical strings. The pattern recognition scheme worked equally well in time and frequency domains for the unlabelled seismograms chosen and results for the intermediate domains were inferior. But the morphology of discrimination can be better studied with the help of Levenshtein triangles and as these triangles are very different for the different domains, the point that the interpolation between time and frequency domain is advantageous is definitely established.
\end{abstract}

Keywords. Syntactic pattern recognition; seismic discrimination; Fourier optics; intermediate spectral domains; pattern primitives; cluster analysis.

\section{Introduction}

Most problems in signal processing (SP) and pattern recognition (PR) are solved in either the original signal domain (time, space or any other) or in the reciprocal domain (frequency, wavenumber or some such similar name). Most frequently, though not universally, the transform used is the Fourier transform (FT). The descriptions in the two reciprocal domains (RD) are equivalent and totally inter-convertible. Moreover, a good deal of theory is developed, so that every unary or binary operation in one domain involving individual signals and their pairs from a class of signals, corresponds to a particular counterpart operation in the RD. Convolution and Schur product constitute such examples for the FT. A pair of counterpart operations implies strict 
equivalence when looked at in any one of the RD, and yet the two operations constituting that pair need not be either of equal computational efficiency or of equal conceptual simplicity. Therefore, it is beneficial to jump back and forth to achieve simplicity of theoretical arguments or computational efficiency.

Inspite of the enormous amount of bipolar literature, bringing out the advantages of the time-domain (or frequency-domain) approach, analysis or techniques, the two reciprocal descriptions are just two viewpoints which need not be or indeed, cannot be, segregated. Jumping back and forth is the simplest recognition of this fact and the procedure is instinctively followed by many. Yet this approach does not amount to a basic attack on the dichotomy of time-domain and frequency-domain approaches. This dichotomy is directly questioned in two ways. One of these leads to using time-frequency distributions (Cohen 1989) or reciprocally conjunctive descriptions (Moharir 1990), the analysis being performed in a domain which has dimensionally reciprocal arguments. The second way is based on the possibility of recognizing the transformation from one domain to the RD not as a jump but as a continuous or at least a multistage journey. It is this approach which is the central theme of this paper.

\section{A clue from Fourier optics}

For the case of FT the concept of a continuum of transformations can be readily established, by drawing some notions from Fourier optics (Van der Lugt 1966).

We begin by defining a quadratic phase function

$$
\psi(x ; f)=\exp \left(j k f x^{2} / 2\right)
$$

where $k=2 \pi / \lambda$ and $\lambda$ is the wavelength of the coherent illumination used. A wavefront may then be denoted by $\psi(x ; d)$, the case of $d=0$ corresponding to the plane wavefront. If $s(x)$ is the amplitude distribution along the $x$ axis of the modulating medium, viz. a transparency, then illuminating it by the wavefront above yields $s(x) \psi(x ; d)$ on its other side. A cylindrical lens with a focal length of $F=1 / f$ introduces a phase $\psi^{*}(x ; f)$, where superscript * denotes complex conjugation. Consider two planes tangential to the lens on the input and output sides. If the amplitude distribution in the former is $s(x)$, that in the latter would be $s(x) \psi^{*}(x ; f)$. Passage through free space by a distance $D=1 / d$ along the optical axis is representable as a convolution of the input amplitude distribution with $d \psi(x ; d)$. Thus the optical set up consisting of lenses, transparencies and illuminating wavefronts can be analysed in terms of the algebra of the $\psi$ functions defined by (1). The rules in that algebra are listed below.

$$
\begin{aligned}
\psi(x ; f) & =\psi^{*}(x ;-f)=\psi(-x ; f), \\
\psi\left(x ; f_{1}\right) \psi\left(x ; f_{2}\right) & =\psi\left(x ; f_{1}+f_{2}\right), \\
\psi(c x ; f) & =\psi\left(x ; c^{2} f\right), \\
\psi(x \pm y ; f) & =\psi(x ; f) \psi(y ; f) \exp ( \pm j k f x y), \\
\lim _{d \rightarrow 0} \psi^{*}(x ; d) & =1, \\
\lim _{d \rightarrow \infty} d \psi(x ; d) & =\delta(x),
\end{aligned}
$$




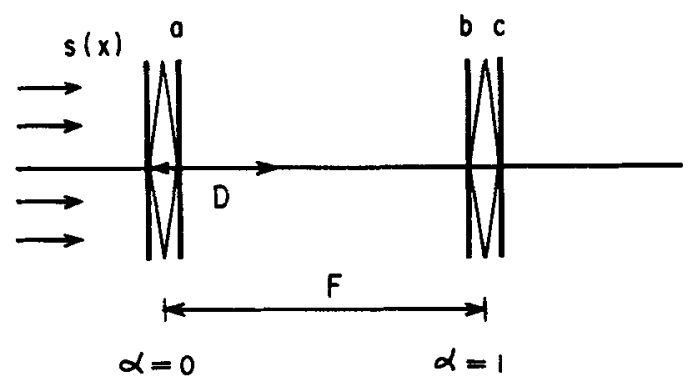

(A)

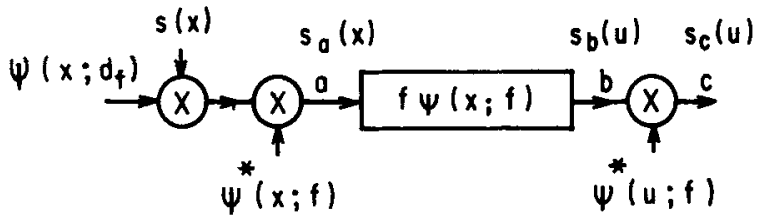

(B)
Figure 1. (a) An optical assembly that can yield a Fourier transform and hence also an interpolation between the time and frequency domains. (b) The representation of the above assembly in the $\psi$-function algebra of Van der Lugt (1966).

$$
\int \psi\left(x ; f_{1}\right) \exp \left(-j k f_{2} x y\right) \mathrm{d} x=\left(c / f_{1}\right) \psi^{*}\left(y ; f_{2}^{2} / f_{1}\right) .
$$

As an illustration consider the optical set up as in figure 1a (Moharir 1975). It is represented mathematically as in figure $1 b$. In particular, let the illumination be by a plane wavefront. Then $\psi\left(x ; d_{1}\right)=1$ as $d_{1}=0$. Then at $a$, we have

$$
s_{a}(x)=s(x) \psi^{*}(x ; f) .
$$

Therefore, at $b$

$$
\begin{aligned}
s_{b}(u) & =\int s_{a}(x) f \psi(u-x ; f) \mathrm{d} x \\
& =f \int s(x) \psi^{*}(x ; f) \psi(x ; f) \psi(u ; f) \exp (-j k f x u) \mathrm{d} x \\
& =f \int s(x) \psi(u, f) \exp (-j k f x u) \mathrm{d} x
\end{aligned}
$$

and hence at $c$

$$
\begin{aligned}
s_{c}(u) & =f \int s(x) \psi(u ; f) \psi^{*}(u ; f) \exp (-j k f x u) \mathrm{d} x \\
& =f \int s(x) \exp (-j k f x u) \mathrm{d} x \\
& =f S(f u / \lambda),
\end{aligned}
$$

which, indeed is the scaled Fourier transform of $s(x)$. 
In between the planes $a$ and $b$, there is a continuum of planes, and in a plane indexed by $\alpha=D / F=f / d$, we have

$$
\begin{aligned}
s_{\alpha}(y) & =d \int s(x) \psi^{*}(x ; f) \psi(y-x ; d) \mathrm{d} x \\
& =d \int s(x) \psi^{*}(x ; f) \psi(y ; d) \psi(x ; d) \exp (-j k d x y) \mathrm{d} x \\
& =d \int s(x) \psi(x ; d-f) \psi(y ; d) \exp (-j k d x y) \mathrm{d} x \\
& =d \psi(y ; d) \int s(x) \psi^{*}(x ; f-d) \exp (-j k d x y) \mathrm{d} x \\
& =(f / \alpha) \psi(y ; f / \alpha) \int s(x) \psi^{*}(x ; f(1-1 / \alpha) \exp (-j k d x y) \mathrm{d} x
\end{aligned}
$$

so that when $\alpha=0$ we get $s_{a}(x)$ of (9) and when $\alpha=1$, we get $s_{b}(u)$ of (10). That is, there is a continuous transition from $s(x) \psi^{*}(x ; f)$ to $f S(f u / \lambda) \psi^{*}(u ;-f)$, i.e. from $s(x)$ subjected to a quadratic phase to its Fourier transform (apart from scaling) subjected to opposite quadratic phase, as $\alpha$ changes from 0 to 1 . For the intermediate values of $\alpha$, the function is as in (12). In particular, in the neutral plane $\alpha=1 / 2$,

$$
s_{n}(y)=2 f \psi(y ; 2 f) \int s(x) \psi^{*}(x ;-f) \exp (-j k 2 f x y) \mathrm{d} x .
$$

Many other optical bench arrangements can be worked out in which there is a continuous transition from a phase-transformed function to its phase-transformed Fourier spectrum.

It is relevant to note that whereas the optical bench provides the facility of performing the relevant transformations instantaneously (not including the time required to make optical transparencies and filters), an optical arrangement achieves the operations in the $\psi$-function algebra only under the thin lens paraxial approximation. The algebra, however, is exact and can be implemented numerically with the desired degree of accuracy. A lens actually would have deletarious effects of chromatic and spherical aberrations, whereas the numerical implementation would be free of these problems, although it would be a victim of finite register length effects. Further, a multi-dimensional extension of these concepts would be restricted optically to only two dimensions, whereas numerically there is no such constraint.

\section{The central idea}

The central idea of this paper will be illustrated with a specific illustration.

Bois $(1980,1982)$ solved the problem of delineating boundaries between different subsurface regimes in the context of search for hydrocarbons using a pattern recognition scheme. The input data consisted of a suite of seismograms, each recorded at a different location below which there might be just a porous rock matrix saturated with water (w), or there might be an oil layer floating on water (wo), or even a gas above water and oil (WOG). All these differences are expected to be reflected in the 
seismograms, the job being to identify them. Individual seismograms were subjected to auto-regressive modelling. In the space of auto-regressive coefficients (ARC), each seismogram was obviously represented by a point, so that the problem was reduced to a geometrical pattern recognition. As one moved along a profile, one could keep track of the distance between the consecutive seismograms, measured in the space of ARC. If the distance abruptly changed, it signified the transition along the profile from one regime of hydrocarbon deposits subsurface to another. We only intend emphasizing here the fact that AR modelling of signals is a useful PR technique. A fuller critique of this approach is available elsewhere (Khattri \& Moharir 1989).

Oldenburg et al (1983) dealt with the problem of estimating acoustic impedances of individual layers from the reflection coefficients obtained by deconvolution of the seismograms. The estimates were unsatisfactory because the reflection coefficients were band-limited due to the narrow band-width of the seismograms. This disadvantage was mitigated by fitting an AR model to the spectrum of a seismogram and using it to extrapolate the spectrum beyond the bandlimit. Notice again that it has been found to be beneficial to subject the spectrum of a seismogram to AR modelling.

There is no logical contradiction in AR modelling being useful for the seismogram as well as its spectrum. Fitting an AR model to a signal is equivalent to expressing its autocorrelation as the weighted sum of complex exponentials. There is no rule against using the same set of basis waveforms in the two RD, though it is somewhat unnatural. In any case, it is a natural question to ask whether a particular set of basis functions would not be most useful for a particular application in a domain in between the two RD. The results in $\S 2$ illustrate that a function and its FT are merely two planes on 'the optical bench', there being a continuum of planes in between. That is, one could call the signal and its Fourier spectrum the identity-spectrum and antipodal spectrum, respectively, there being a whole spectrum of spectra in between, indexed by $\alpha$. It need not be then, that any PR strategy works the best in either the identity-spectral or antipodal-spectral domain or that the choice is restricted to these dichotomous spectral domains. One could experiment whether some intermediate $\alpha$-spectral domain would give the best results for a particular application and a specific PR strategy. Such a question makes sense because PR strategies are normally ad hoc exploratory devices and are not optimized in the context of prescribed desiderata and signal specifications.

The next two sections deal with such an experiment.

\section{Pattern recognition scheme}

The context chosen was that of seismic discrimination. From the seismogram it was intended to figure out whether the subsurface strata below the station of recording were (a) gas, oil and water bearing (WOG), (b) only oil and water bearing (wo) or (c) only water bearing $(w)$.

Many synthetic seismograms corresponding to the three situations above were generated. The algorithm of Khattri et al (1978) was used for the purpose. The algorithm gives the spectrum of the synthetic seismogram first.

Syntactic pattern recognition was used as it is generally believed now that it has richer potential than that of the statistical or decision-theoretic approach. The basic idea of this approach is that a complex pattern is built recursively upwards anabolically from simpler pattern primitives. This procedure is analogous to making words from 
letters, phrases, clauses and sentences from words etc. The PR procedure then is to parse the complex pattern using the rules of anabolic composition as grammar ( $\mathrm{Fu}$ 1974; Pavlidis 1977; Fu and Liu 1983).

Specifically, AR coefficients were used as pattern primitives as they have some physical meaning from a seismic viewpoint (Silvia and Robinson 1978). A seismogram was segmented and an AR model fitted to every segment. In the space of AR coefficients, clusters were identified using a modified $K$-means clustering algorithm (Wilpon $\&$ Rabiner 1985). Every cluster was used as an individual letter in an alphabet, the size of which was the number of clusters. Thus, any seismogram (or any of its $\alpha$-spectra) is represented as a string of letters.

In a learning phase the characteristic string for each of the three hydrocarbonbearing situations above is identified. The unlabelled seismogram characterized by a string is identified with the class from the characteristic string of which it is the closest. The distance measure used for this purpose is the Levenshtein distance (Levenshtein 1966) which is defined for alphabetical strings of unequal lengths. In our case, the segmentation strategy for seismograms was not adaptive. Therefore, the strings to be compared are all of the same length and the distance measure is just the sum of distances between the corresponding letters in the strings compared. The distance between letters was defined as the distance between the centroids of the clusters in the space of ARC defining them. The distances between letters can induce distances between strings in other ways also (Moharir 1988, pp. 1-7).

The optical arrangement used for interpolation between the two RD was as shown in figure 2. In keeping with the comments at the end of $\$ 2$, the entire 'optics' was replaced by the relevant $\psi$-function algebra which was performed on a digital computer, numerically. The signal transparency $(s(x))$ is at a distance $2 F$ in front of the lens and is illuminated by a plane wavefront. The image is obtained in a plane at a distance $2 F$ behind the lens in which

$$
s_{b}(y)=(f / 2) s(-y) \psi(y ; 3 f / 2),
$$

and the FT is obtained in a plane at a distance $F$ behind the lens in which

$$
s_{a}(x)=(f / 2) S(f x / 2 \lambda) \psi(x ;-f) .
$$

Thus again, apart from scaling, there is a transformation from the phase-modified FT $s_{a}(x)$ to its phase-modified time-domain counterpart $s_{b}(y)$ by propagation through

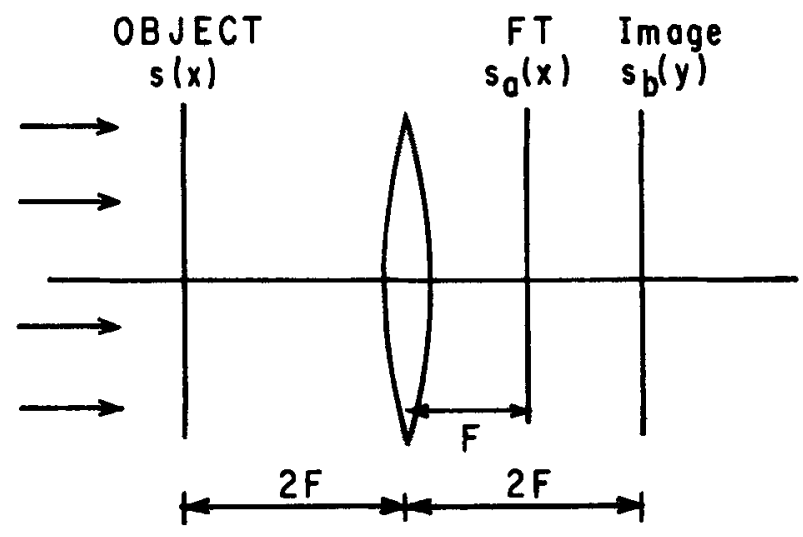

Figure 2 Another optical assembly that yields the image and the Fourier transform separated by a focal length of the lens. This can also be used for interpolation between the two reciprocal domains. 
free-space which is represented by a convolution. We can use the $\alpha$-spectrum in any intermediate plane between $a$ and $b$ for which the distance behind the lens is $(2-\alpha) F$, $\alpha=0$ and 1 corresponding to phase-modified identity-spectrum and antipodalspectrum. This arrangement is more convenient than that of figure la because the algorithm used for generating synthetic seismograms yields the Fourier spectra more readily than the seismograms themselves.

\section{Results}

The optimum number of clusters in the FT domain $(\alpha=1)$ was found to be 7 . For all other $\alpha$-spectral domains the optimal number of clusters was 6. The values of $\alpha$ used were $\alpha=0,0.25,0.5,0.75$ and 1 . Table 1 (Chhibber 1988) shows the alphabet for each

Table 1. Definition of the alphabet for various $\alpha$-spectral domains.

\begin{tabular}{|c|c|c|c|}
\hline \multirow[b]{2}{*}{$\alpha$} & \multirow{2}{*}{$\begin{array}{c}\text { Cluster } \\
\text { denoted by }\end{array}$} & \multicolumn{2}{|c|}{$\begin{array}{l}\text { Pattern primitives or the } \\
\text { normalized AR coefficients } \\
\text { of the cluster centroid }\end{array}$} \\
\hline & & First third & Second/third \\
\hline 0 (time) & $\begin{array}{l}\text { A } \\
B \\
C \\
D \\
E \\
F\end{array}$ & $\begin{array}{r}-1.77212 \\
0.85127 \\
-0.09511 \\
-0.68313 \\
-2.78341 \\
-1.98423\end{array}$ & $\begin{array}{r}0.09575 \\
-0.59566 \\
-0.19318 \\
-0.61351 \\
-0.38632 \\
-0.03394\end{array}$ \\
\hline 0.25 & $\begin{array}{l}\text { A } \\
\text { B } \\
\text { C } \\
\text { D } \\
\text { E } \\
\text { F }\end{array}$ & $\begin{array}{r}-1.22151 \\
3.00732 \\
-2.51561 \\
-0.98653 \\
-0.58241 \\
-0.95511\end{array}$ & $\begin{array}{r}0.81855 \\
1.19734 \\
-0.58312 \\
0.07318 \\
0.51332 \\
0.63415\end{array}$ \\
\hline $0-50$ & $\begin{array}{l}\text { A } \\
\text { B } \\
\text { C } \\
\dot{D} \\
\text { E } \\
\text { F }\end{array}$ & $\begin{array}{r}3.33416 \\
1.67781 \\
2 \cdot 76851 \\
1.31377 \\
1.63376 \\
1.00813\end{array}$ & $\begin{array}{r}0.67685 \\
0.51319 \\
0.59133 \\
0.12139 \\
0.14133 \\
-0.13319\end{array}$ \\
\hline 0.75 & $\begin{array}{l}\text { A } \\
\text { B } \\
\text { C } \\
\text { D } \\
\text { E } \\
\text { F }\end{array}$ & $\begin{array}{r}0.10438 \\
1.89351 \\
1.25781 \\
-1.30047 \\
-0.56372 \\
-2.40197\end{array}$ & $\begin{array}{r}0.21679 \\
0.44862 \\
-0.46389 \\
0.32153 \\
-0.15682 \\
-0.58831\end{array}$ \\
\hline $1(\mathrm{FT})$ & $\begin{array}{l}\text { A } \\
\text { B } \\
\text { C } \\
\text { D } \\
\text { E } \\
\text { F } \\
\text { G }\end{array}$ & $\begin{array}{r}3.09856 \\
2.83647 \\
-2.63741 \\
-1.37582 \\
-2.49361 \\
0.92741 \\
-1.43772\end{array}$ & $\begin{array}{r}-0.42567 \\
1.24869 \\
0.52718 \\
1.07452 \\
-0.58435 \\
0.83511 \\
0.86792\end{array}$ \\
\hline
\end{tabular}


one of these domains. An AR model of order 3 was used and the two pattern primitives chosen were $\alpha_{31} / \alpha_{33}$ and $\alpha_{32} / \alpha_{33}$ where $\alpha_{r j}$ is the $j$ th AR coefficient in an AR model of order $r$. AR(3) model is hormally found adequate in seismic discrimination work (see e.g. Bois 1980, 1982; Kapil et al 1985). Formally, there are many criteria to choose the order of the so-called optimal AR model (Akaike 1969, 1970, 1972-1974; Ishii \& Suzumura 1977; Ishii et al 1978) which patently give different estimates for the order and leave much to be desired (see e.g. Gutowski et al 1978, Nitzberg 1979). It does come to exercising subjective judgment, even if cloaked under the impression of objectivity created by an impressive nomenclature for the criterion selected, as the question of why that particular criterion is preferred over others proposed is still unanswered.

Table 2. Distances between pairs of letters of an alphabet for various $\alpha$-spectral domains.

\begin{tabular}{|c|c|c|c|c|c|c|c|}
\hline & $\mathbf{A}$ & B & C & D & $\mathbf{E}$ & $\mathbf{F}$ & G \\
\hline \multicolumn{8}{|c|}{$\alpha=0$ (time domain) } \\
\hline $\mathbf{A}$ & 0.00 & & & & & & \\
\hline B & 0.63 & 0.00 & & & & & \\
\hline C & 0.20 & $0-43$ & 0.00 & & & & \\
\hline D & 0.53 & 0.10 & $0-34$ & 0.00 & & & \\
\hline E & 0.37 & 1.00 & 0.57 & 0.90 & 0.00 & & \\
\hline $\mathbf{F}$ & $0-09$ & 0.72 & 0.28 & 0.62 & 0.28 & 0.00 & \\
\hline \multicolumn{8}{|c|}{$\alpha=0.25$} \\
\hline A & 0.00 & & & & & & \\
\hline B & 0.58 & 0.00 & & & & & \\
\hline C & 0.60 & 1.00 & 0,00 & & & & \\
\hline D & 0.31 & 0.62 & $0-38$ & 0,00 & & & \\
\hline E & 0.12 & 0.53 & 0.50 & 0.16 & 0.00 & & \\
\hline $\mathbf{F}$ & 0.09 & $0-56$ & $0-55$ & 0.22 & 0.06 & 0.00 & \\
\hline \multicolumn{8}{|c|}{$\alpha=0.5$} \\
\hline $\mathbf{A}$ & 000 & & & & & & \\
\hline B & 0.44 & 0.00 & & & & & \\
\hline C & 0.20 & 0.24 & 0.00 & & & & \\
\hline D & $0-87$ & 0.43 & 0.67 & 0.00 & & & \\
\hline E & 0.69 & 0.25 & $0-49$ & 0.18 & 0.00 & & \\
\hline F & 1.00 & 0.56 & $0-80$ & 0.13 & 0.31 & $0-00$ & \\
\hline \multicolumn{8}{|c|}{$\alpha=0.75$} \\
\hline $\mathbf{A}$ & $0-00$ & & & & & & \\
\hline B & 0.37 & 0.00 & & & & & \\
\hline C & 0.36 & 0.47 & $0-00$ & & & & \\
\hline D & 0.19 & 0.53 & 0.53 & 0,00 & & & \\
\hline $\mathbf{E}$ & 0.20 & 0.53 & $0-30$ & 0.28 & 0,00 & & \\
\hline $\mathbf{F}$ & 0.63 & 1.00 & 0.60 & 0.63 & 0.43 & 000 & \\
\hline \multicolumn{8}{|c|}{$\alpha=1$ (FT domain) } \\
\hline A & $0-00$ & & & & & & \\
\hline B & 0.99 & 0.00 & & & & & \\
\hline C & 0.74 & 0.68 & $0-00$ & & & & \\
\hline D & 0.78 & 0.38 & $0-30$ & 0.00 & & & \\
\hline $\mathbf{E}$ & 0.70 & 100 & 0.33 & $0-62$ & $0-00$ & & \\
\hline$F$ & 0.84 & 0.17 & 0.53 & 0.23 & $0-83$ & $0-00$ & \\
\hline G & 0.76 & 0.32 & 0.38 & 0.09 & 0.68 & 0.15 & 000 \\
\hline
\end{tabular}


Table 3. Characteristic strings for the three hydrocarbon regimes for all the $\alpha$-spectral domains.

\begin{tabular}{lccc}
\hline & \multicolumn{3}{c}{ Hydrocarbon regime } \\
\cline { 2 - 4 }$\alpha$ & $\begin{array}{c}\text { Gas, oil and water } \\
\text { (woG) }\end{array}$ & $\begin{array}{c}\text { Oil and water } \\
\text { (wo) }\end{array}$ & $\begin{array}{c}\text { Water } \\
(w)\end{array}$ \\
\hline 0.00 & EA & AC & DD \\
0.25 & AF & BC & BD \\
0.50 & AD & BE & BD \\
0.75 & EA & EE & EC \\
1.00 & BC & DG & FA \\
\hline
\end{tabular}

Distances between pairs of letters are shown in table 2 (Chhibber 1988) for various $\alpha$-spectral domains. The largest distance is normalized to unity in every case.

In all, 105 seismograms comprising 7 anticlinal models were used for learning. These models spanned nearly the whole range of seismic parameters, viz. velocity, density and attenuation. Table 3 (Chhibber 1988) shows the characteristic strings for all the $\alpha$-spectral domains. For simplicity the spectral sequence in every $\alpha$-spectral domain was segmented in only two parts.

In all, 15 unlabelled seismograms were subjected to classification based on the nearest neighbour rule in terms of the Levenshtein distance. In the time $(\alpha=0)$ and frequency $(\alpha=1)$ domains 2 of these were misclassified. In the misclassified cases, the thicknesses of the oil and gas layers were on the thresholds of seismic resolution. This signifies that the syntactic pattern recognition approach outlined above can be useful in seismic discrimination of hydrocarbon deposits. This is a worthwhile indication in itself. For the intermediate $\alpha$-spectral domains $\alpha=0.25,0.50$ and 0.75 , the numbers of misclassified seismograms were 4,6 and 8 respectively. In some of the misclassified cases the thicknesses of oil and gas layers were above the thresholds of seismic discrimination.

It is necessary to investigate to what extent these results are biased by the choice of unlabelled synthetic seismograms. For that purpose, the Levenshtein distances between the pairs of characteristic strings representing the hydrocarbon situations w, wo and woG are calculated with the help of tables 2 and 3 and the Levenshtein triangles are plotted in figure 3 . The Levenshtein distances are shown by the sides. Note that the morphology of these discrimination triangles is very different for every $\alpha$-spectral domain. The relative sizes of these triangles are an artifact of the procedure used to normalize the distances between letters and hence could be ignored. The discrimination potentials do not depend on the size of the triangle but depend on its shape. The best discrimination is possible between (a) $w$ and woG for $\alpha=0$ and 0.75 , (b) wo and woG for $\alpha=0.25$ and 0.50 , and (c) $w$ and wo for $\alpha=1$. Thus it is clearly established that under certain circumstances it may be advantageous to work in intermediate $\alpha$-spectral domains and their inferior performance inferred earlier was a biased judgement. Moreover, for optimal results, the entire PR task need not be performed in the same $\alpha$-spectral domain. To illustrate this point, let the classification be performed not by the nearest neighbour rule, but by eliminating the farthest neighbour first. Let us be working in the $\alpha=0$ domain and let the eliminated option be $w$. Then the retained options are wo and woG and as they are much better discriminated in the $\alpha=0.25$ domain rather than in the $\alpha=0$ domain, it would make sense to shift to the $\alpha=0.25$ domain for the residual task. 
WOG
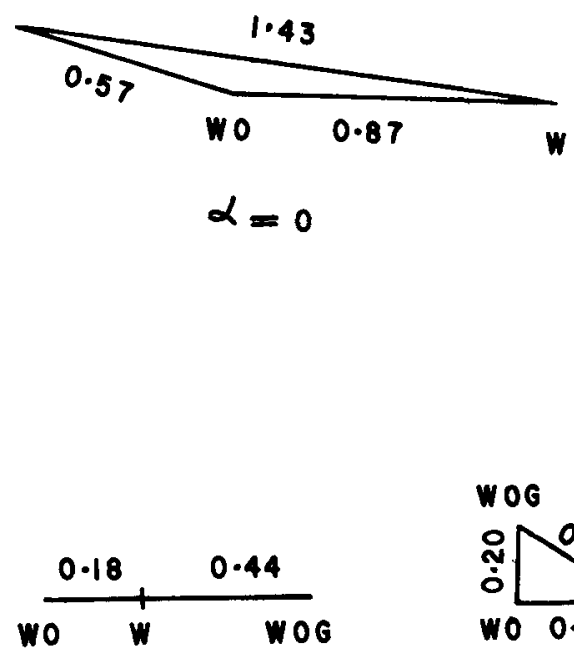

$$
\alpha=0.5
$$

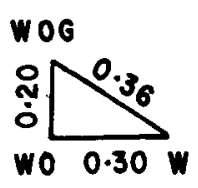

$\alpha=0.75$

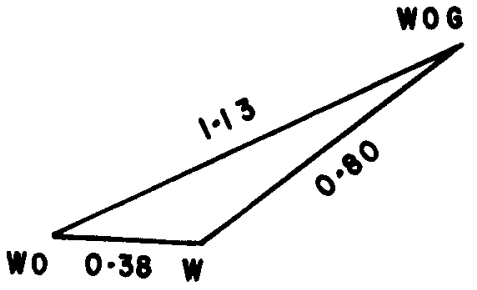

$\alpha=0.25$

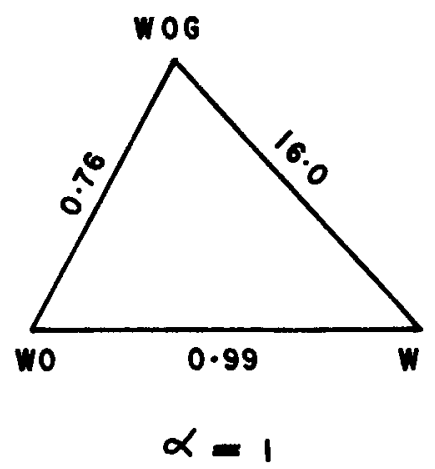

Figure 3. Levenshtein triangles for the seismic discrimination among three hydrocarbon situations viz. w (water only), wo (water and oil), woG (water, oil and gas) for five $\alpha$-spectral planes using the optical set-up of figure 2.

\section{Conclusion}

Two Fourier-optical arrangements which achieve transformation from the quadratic phase-modified signal to the quadratic phase-modified FT over a distance $F$ by propagation through free space are discussed. In any of them there is a continuous transformation from one domain to its RD and therefore a possibility of interpolation between those two reciprocal domains. A simple idea that any PR algorithm for a specific problem could be implemented in any of the intermediate spectral domains has been put forward.

An experiment performed with a simple syntactic PR scheme did effectively make a point that in some situations better results could be obtained in one of the intermediate $\alpha$-spectral domains than in either the time- or the frequency-domain. It also illustrated that an adaptive journey among various $\alpha$-spectral domains may be desirable.

This work could be viewed as an extension of that by Moharir (1990, pp. 11-20) as the basic motivation of both papers is to step out of the dichotomy of time- and frequency-domain approaches. In the earlier paper it has been pointed out that Wigner distribution (Wigner 1932; Cohen 1989) and ambiguity function in radar (Cook \& Bernfeld 1967, pp. 1-15, 59-108, 226-295; Vakman 1968) also form an FT pair and hence are descriptions in 2-dimensional RD. Therefore, in an optical set-up one can have a continuous transition from one of them to the other after generating one optically. This point has already been recognized (Saleh 1979; Bartelt et al 1980; Bamler \& Glunder 1983; Ojeda-Castaneda \& Sicre 1984; Gupta \& Askura 1986). Then in the spirit of the central notion of this paper there could be an interpolation between 
the two reciprocally conjunctive descriptions, namely, the Wigner distribution of a signal and the ambiguity function. Both of these could be replaced by their generalizations without destroying their link as descriptions in RD. Working in some intermediate interpolatory domain may, in some cases at least, yield better results.

Results obtained in the time and frequency domains hint at the utility of a syntactic pattern recognition approach for seismic discrimination. One of the least formalized parts of the PR scheme is the choice of the pattern primitives. The other results illustrated by figure 3 could mean that this paper has extended the repertoire of pattern primitives. Pattern primitives could be any set of parameters, such as ARC, and moreover, they could be extracted from any $\alpha$-spectral domain depending on contingencies. That is, there could be an a priori choice of a PR strategy and the spectrum of spectra could be scanned for the optimum results with it.

It is a short step from here to use the spectrum of spectra even to define patterns. A pattern is a local concentration of energy in a few pockets in some domain. The spectrum of spectra may be scanned to identify such a localization of energy in some $\alpha$-spectral domain. The pattern then is characterized by the specification of that $\alpha$ and the description of the energy localization.

The comments made by Prof D Guptasarma of the National Geophysical Research Institute on an earlier presentation of these ideas have been useful. Thanks are also due to Prof V U Reddy for his invitation to write this paper.

\section{References}

Akaike H 1969 Power spectrum estimation through autoregressive model fitting. Ann. Inst. Stat. Math. 21: $407-419$

Akaike H 1970 Statistical predictor identification. Ann. Inst. Stat. Math. 22: 203-217

Akaike $H 1972$ Information theory and an extension of the maximum likelihood principle. Proc. 2 nd Int. Symp. on Info. Theory

Akaike H 1973 Maximum likelihood identification of Gaussian autoregressive moving average models. Biometrika 60: 255-265

Akaike H 1974 A new look at statistical model identification. IEEE Trans. Autom. Control AC-19: 716-723

Bamler R, Glunder H 1983 The Wigner distribution function of two-dimensional signals coherent -optical generation and display. Opt. Acta 30: 1789-1803

Bartelt H O, Brenner K-H, Lohmann W 1980 The Wigner distribution function and its optical production. Opt. Commun. 32: 32-38

Bois P 1980 Autoregressive pattern recognition applied to the delimitation of oil and gas reservoirs. Geophys. Prospect. 28: 572-591

Bois P 1982 Some comments on the applications of pattern recognition to oil and gas exploration. Geoexploration 20: 147-159

Chhibber R 1988 Direct detection of subsurface hydrocarbons by syntactic pattern recognition of seismic signals, $\mathrm{M}$ Tech dissertation, Roorkee University, Roorkee

Cohen L 1989 Time-frequency distributions - a review. Proc. IEEE 77: 941-981

Cook C E, Bernfeld M 1967 Radar signals: An introduction to theory and application (New York: Academic Press)

Fu K S 1974 Syntactic methods in pattern recognition (New York: Academic Press)

Fu K S, Liu H H 1983 An application of syntactic pattern recognition to seismic discrimination. IEEE Trans. Geosci. Electron. GE-21: 125-132 
Kapil S L, Moharir P S, Khattri K N 1985 BAR-ARMA predictive deconvolution of seismograms. J. Assoc. Explor. Geophys. 6: 1-6

Gupta A K, Askura T 1986 New optical system for the efficient display of Wigner distribution functions using a single object transparency. Opt. Commun. 60: 265-268

Gutowski R R, Robinson E A, Trietel S 1978 Spectral estimation; fact or fiction. IEEE Trans. Geosci. Electron. GE-16: 80-84

Ishii N, Iwata A, Suzumura N 1978 Evaluation of an autoregressive process by information measure. Int. J. Syst. Sci. 9: 743-752

Ishii N, Suzumura N 1977 Estimation of the order of autoregressive process. Int. J. Syst. Sci. 8: 905-914

Khattri K N, Gaur V K, Mithal R, Tandon A K 1978 Seismogram synthesis in multilayered dissipative media. Geoexploration 16: 185-202

Khattri K N, Moharir P S 1989 Pattern recognition techniques for seismic detection of hydrocarbon deposits. Indian J. Geol. 61: 243-271

Levenshtein 1966 Binary codes capable of correcting deletions, insertions and reversals, Sov. Phys. Dokl. (Engl. Transl.) 10: 707-710

Moharir P S 1975 Normalized optical filtering. Indian J. Pure Appl. Phys. 13: 836-839

Moharir P S 1988 Multilingual pattern recognition for geoexploration. In Frontiers in exploration geophysics (ed.) B B Bhattacharya (New Delhi: Oxford \& IBH)

Moharir P S 1990 Reciprocally conjunctive descriptions of signals. In Signal processing. communications and networking (eds) V U Reddy, A Paulraj (New Delhi: Tata McGraw-Hill)

Nitzberg R 1979 Spectral estimation: an impossibility? Proc. IEEE 67: 437-438

Ojeda-Castaneda J, Sicre E E 1984 Bilinear optical systems, Wigner distribution function and ambiguity function representations. Opt. Acta 31: 255-260

Oldenburg D W, Scheuer T, Levy S 1983 Recovery of the acoustic impedance from reflection seismograms. Geophysics 48: 1318-1337

Pavlidis T 1977 Structural pattern recognition (Berlin: Springer-Verlag)

Saleh B E A 1979 Optical bilinear transformations - general properties. Opt. Acta 26: 777-799

Silvia M T and Robinson E A 1978 Use of kepstrum in signal analysis. Geoexploration 16: 55-73

Vakman D E 1968 Sophisticated signals and uncertainty principle in radar (Berlin: Springer-Verlag)

Van der Lugt A 1966 Operational notation for the analysis and systhesis of optical data-processing systems. Proc. IEEE 54: 1055-1063

Wigner E P 1932 On the quantum correction for thermodynamic equilibrium. Phys. Rev. 40: 749-759

Wilpon J G, Rabiner L R 1985 A modified K-means clustering algorithm for use in isolated word recognition. Trans. IEEE Acoust., Speech Signal Process. ASSP-33: 587-594 